\title{
An Active Matrix Flat Panel Dosimeter (AMFPD) for in-phantom dosimetric measurements
}

\author{
Jean M. Moran, ${ }^{\text {a) }}$ Donald A. Roberts, Teamour S. Nurushev, Larry E. Antonuk, \\ Youcef El-Mohri, and Benedick A. Fraass \\ Department of Radiation Oncology, University of Michigan, Ann Arbor, Michigan, 48109-0010
}

(Received 7 September 2004; revised 24 November 2004; accepted for publication 11 December 2004; published 25 January 2005)

\begin{abstract}
An $a$-Si Active Matrix Flat Panel Imager (AMFPI) prototype developed in-house has been modified to function as an in-phantom dosimetry system providing high resolution two-dimensional (2-D) data. This Active Matrix Flat Panel Dosimeter (AMFPD) system can be used as a replacement device for standard in-phantom dosimeters, such as scanning ion chambers in water, or film in solid water. The initial characterization of the device demonstrates a wide dynamic range (up to 160 cGy), a stable calibration curve (less than $1.5 \%$ variation over 1 year), dose rate independence (less than $1 \%$ ), and excellent agreement of output factors with ion chamber measurements for a range of field sizes (less than 2\%). The device also compares well to film for 2-D planar dose distributions. It is expected that the AMFPD system will be useful for beam commissioning, algorithm verification test data, and routine IMRT quality assurance dosimetry. (C) 2005 American Association of Physicists in Medicine. [DOI: 10.1118/1.1855012]
\end{abstract}

Key words: dosimetry, amorphous silicon, active matrix flat panel dosimeter, quality assurance

\section{INTRODUCTION}

The quality assurance of linear accelerators and treatment planning systems requires the acquisition of data in three dimensions. The development of electronic portal imaging systems has resulted in an increased interest in using such systems for dosimetric applications to replace 2-D film measurements. CCD cameras with fluorescent screens, ${ }^{1-3}$ liquidfilled ion chamber arrays, ${ }^{4}$ and amorphous silicon flat panel imagers have been investigated. ${ }^{5-7}$ These systems have been applied to transit dosimetry ${ }^{8-11}$ and pre-treatment field verification. ${ }^{7,12,13}$

Previous work by El-Mohri ${ }^{14}$ involved an examination of in-phantom dosimetric properties of a prototype active matrix flat panel imager (AMFPI) operated in an indirect mode (with a fluorescent screen) and in a direct mode (with no screen present). The detector response was evaluated as a function of dose, dose rate, and stability of the pixel response for doses up to approximately $25 \mathrm{cGy}$. Comparisons were made to ion chamber measurements in water. The study demonstrated that the AMFPI, when operated in the direct detection mode, had a response much more similar to the measured ion chamber data than when operated in an indirect detection mode. This result suggested that it would be possible to obtain dose distribution information from an active matrix flat panel detector (AMFPD) operated in a direct detection mode.

The current work extends that of the previous investigation ${ }^{14}$ by modifying an in-house developed AMPFI device, similar to one used in previous work, for higher dose applications. The system response was investigated over a wider range of doses such as those required for commissioning and patient dosimetric measurements of static and IMRT fields. The system was designed specifically to replace film in water-equivalent phantoms.

\section{MATERIALS AND METHODS}

\section{A. System description}

The $a$-Si detector array has $512 \times 512$ pixels with a 508 $\mu \mathrm{m}$ pixel pitch, yielding a detector area of approximately $26 \times 26 \mathrm{~cm}^{2}$. To increase the dynamic range of the detector, the image acquisition electronics for the system were modified from the original AMFPI to incorporate preamplifiers whose gains were matched to the full pixel charge capacity of approximately $90 \mathrm{pC}$ at a bias of $-5 \mathrm{~V}$ applied to the photodiodes $\left(V_{\text {bias }}\right)$. The AMFPD is operated in the direct detection mode. ${ }^{14}$ These changes result in a reduction of the overall system gain.

\section{B. Operating conditions}

The array was mounted in an aluminum support frame (see Fig. 1). During measurements, the system is operated in the dark and covered with a dark cloth to minimize any ambient room light. A $2 \mathrm{~cm}$ thick piece of $30 \times 30 \mathrm{~cm}^{2}$ solid water (Gammex-RMI 457, Middleton, WI) is placed directly on the array. The weight of additional $40 \times 40 \mathrm{~cm}^{2}$ solid water pieces was borne by the aluminum support frame in order to protect the array. This setup results in an air gap of less than $0.5 \mathrm{~mm}$ between the solid water pieces. A $6.5 \mathrm{~cm}$ thick slab of solid water is placed below the detector to provide adequate backscatter. The system was operated in the radiographic mode in which the pixel data are read out after the complete dose delivery.

A number of steps are followed to determine the measured dose with the AMFPD system. Dark frame measurements are made to extract the dark current and individual pixel offsets using a linear model. The measured dose for an irradiated frame is determined in two steps. First, the dark 


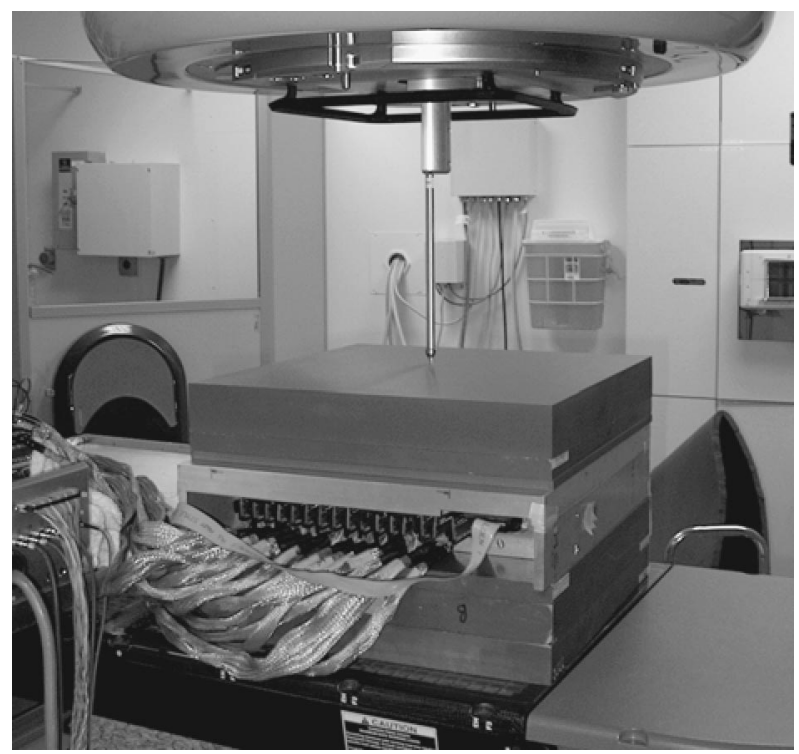

FIG. 1. Active Matrix Flat Panel Dosimeter mounted in a support frame and positioned in solid water phantom.

current and offsets for a given irradiation are removed from the measurement to obtain a corrected measurement $\left(M_{\text {corr }}\right)$ at each pixel position:

$$
M_{\operatorname{corr}_{i, j}}=M_{\text {raw }_{i, j}}-I_{\mathrm{dark}_{i, j}} \cdot \Delta t-O_{i, j},
$$

where, for a given pixel $(i, j), M_{\text {raw }}$ is the raw or uncorrected radiation image, $I_{\text {dark }}$ is the dark current, $\Delta t$ is the frame time for the dark image, and $O$ is the offset correction. The dose is calculated from

$$
D_{i, j}=f\left(G_{i, j} \cdot M_{\text {corr }_{i, j}}\right),
$$

where, for each pixel $(i, j), D$ is the dose, $G$ is the gain correction, and $M_{\text {corr }}$ is the corrected measurement (in corrected ADC units). The function, $f$, is used to convert from corrected ADC units to dose using a dose calibration curve. The dose calibration curve is determined from a high tension spline applied to the measured calibration data. The splined fit to the data is used as a lookup table to determine the dose at each pixel position.

Because the pixel gains are sensitive to the charge trapping density, the system is operated in a specific sequence. At the beginning of each set of measurements, the system is initialized with a $30 \times 30 \mathrm{~cm}^{2}$ flood field for a dose of approximately $240 \mathrm{cGy}$. The electronics are moved as far as possible from the detector so they are not directly exposed. Prior to the delivery of each measurement field, another 30 $\times 30 \mathrm{~cm}^{2}$ flood field (approximately $80 \mathrm{cGy}$ ) is delivered so that each pixel has approximately the same charge trapping density prior to the beginning of each measurement. For each pixel, the gain stability was checked by comparing the flood field to measurements made with film (corrected to dose) for the same field size and depth in solid water. Any data from bad address lines or malfunctioning preamplifier channels are removed and replaced with values determined from the application of a 3 pixel $\times 3$ pixel median filter. Note that the median filter is only used to correct values for bad pixels; pixels with a normal response are not smoothed in any way.

\section{Device characterization}

The AMFPD response was evaluated as a function of the dose, dose rate, beam energy, and field size. In addition, measurements of the $6 \mathrm{MV}$ dosimetric calibration curve were made one year apart to evaluate the long-term stability of the calibration. All measurements with the AMFPD and film were made perpendicular to the beam.

Prior to use of the system for dosimetric measurements, the response of each preamplifier channel was determined by injecting known charges into each channel. The stability of the preamplifier calibration was also evaluated over time by evaluating flood field measurements made over a period of 1 year. To assess the long-term stability, the charge injection was repeated for a single channel in each preamplifier.

The initial characterization of the AMFPD was performed on a linear accelerator using $6 \mathrm{MV}$ and $16 \mathrm{MV}$ photon beams equipped with a 120 leaf multileaf collimator (Varian $21 \mathrm{EX}$, Varian Medical Systems, Palo Alto, CA). Unless explicitly stated, experiments were conducted at $90 \mathrm{~cm}$ SSD, $10 \mathrm{~cm}$ depth, and a dose rate of $320 \mathrm{cGy} / \mathrm{min}$. The linear accelerator was calibrated so that 1 monitor unit (MU) with a 10 $\times 10 \mathrm{~cm}^{2}$ jaw field results in a dose of $0.8 \mathrm{cGy}$ on the central axis at $10 \mathrm{~cm}$ depth and $90 \mathrm{~cm}$ SSD.

For 6 and $16 \mathrm{MV}$, a dose characterization curve was measured for doses ranging from 0.8-160 cGy under the accelerator calibration conditions. The curve was measured again one year later for $6 \mathrm{MV}$ to assess the long-term stability of the system. The AMPFD response was also measured at 80 cGy (100 MU) for dose rates of 80 to $480 \mathrm{cGy} / \mathrm{min}$ in increments of $80 \mathrm{cGy} / \mathrm{min}$ (100 to $600 \mathrm{in} \mathrm{MU/min).} \mathrm{For} \mathrm{this}$ analysis, the AMFPD response was averaged over a detector region of $11 \times 11$ pixels at the center of the field. The dependence of the pixel response on the beam energies of 6 and 16 MV was investigated under the calibration conditions for a detector region of $6 \times 6$ pixels at the center of the field.

To assess the field size dependence of the system, measurements were made for a $6 \mathrm{MV}$ beam for MLC fields ranging from $3 \times 3 \mathrm{~cm}^{2}$ to $25 \times 25 \mathrm{~cm}^{2}$ at $90 \mathrm{~cm} \mathrm{SSD}$ and $10 \mathrm{~cm}$ depth. To demonstrate the potential of the system, AMFPD planar dose distributions were compared to film for a 10 $\times 10 \mathrm{~cm}^{2}$, an MLC-shaped static field and a sample IMRT head and neck field.

The lag and ghosting effects associated with charge trapping at high doses for this device, when operated under similar conditions as described in this work, have previously been reported. ${ }^{15}$

\section{RESULTS}

Long-term stability was evaluated for the preamplifier calibration and the system. As noted above, the response of each preamplifier channel was determined by injecting known charges into each channel. At 1.5 years later, the response of a single channel for each preamplifier was reevaluated using the charge injection method [Fig. 2]. The 


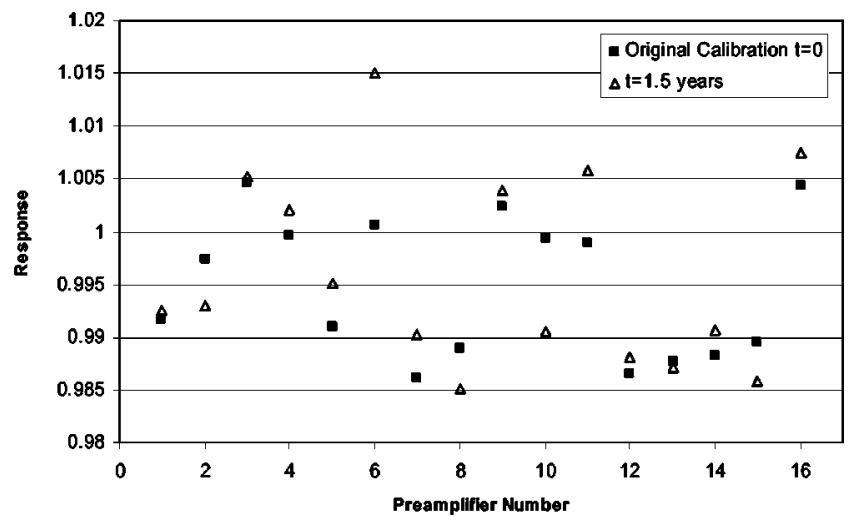

FIG. 2. The response for a single channel in each preamplifier is shown at two time points ( 1.5 years apart). The variation in the response at the two time points was less than $1.5 \%$.

variation in the response over that time was found to be less than $1.5 \%$. The stability of the dosimetric response of the system was evaluated by measurement of the dose calibration curve at an initial time point and at 1 year later for 6 MV. The calibration curve, derived using Eq. (1), was measured for doses ranging from 0.8 to $160 \mathrm{cGy}$ [Fig. 3(a)]. The system response varied by less than $1.5 \%$ over that time [Fig. 3(b)].
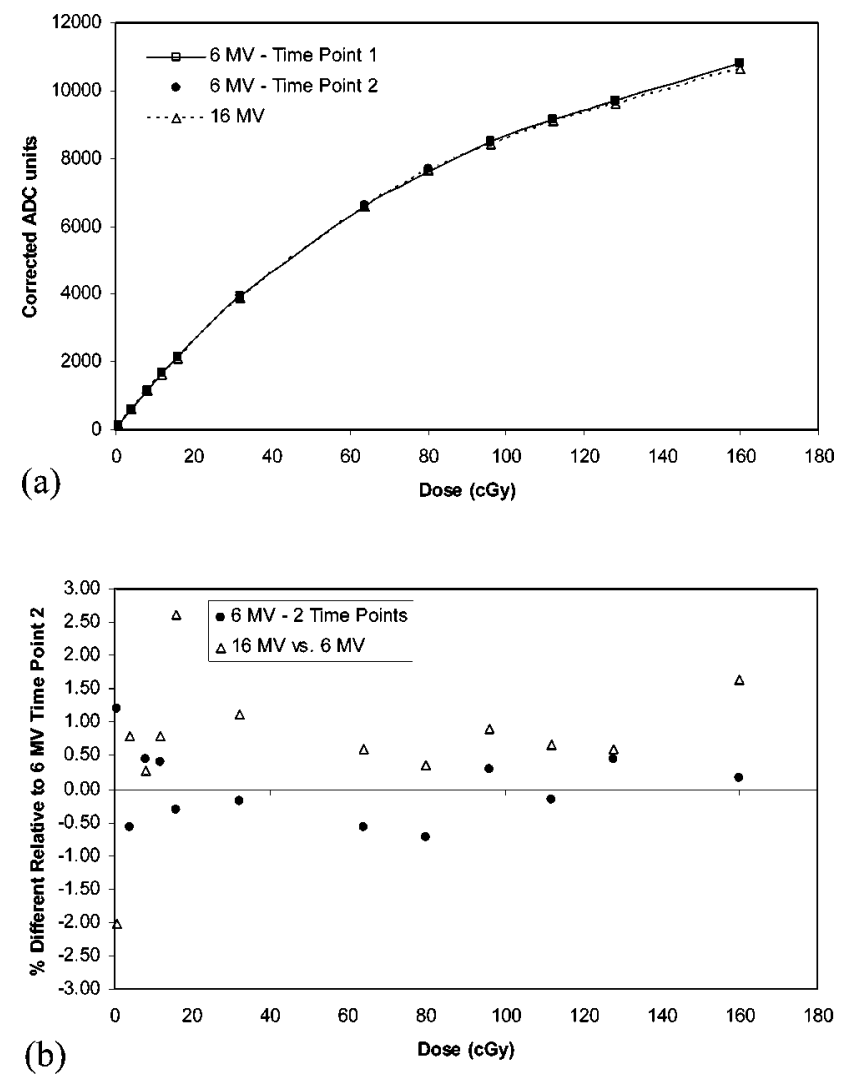

FIG. 3. (a) AMFPD dose calibration curve for 6 MV photons at 10 $\times 10 \mathrm{~cm}^{2}$ at $10 \mathrm{~cm}$ depth at 2 different times measured 1 year apart (solid line with squares and circles) and for $16 \mathrm{MV}$ photons (dashed line with triangles). (b) Percent difference between $6 \mathrm{MV}$ data points and $6 \mathrm{MV}$ and $16 \mathrm{MV}$ values.
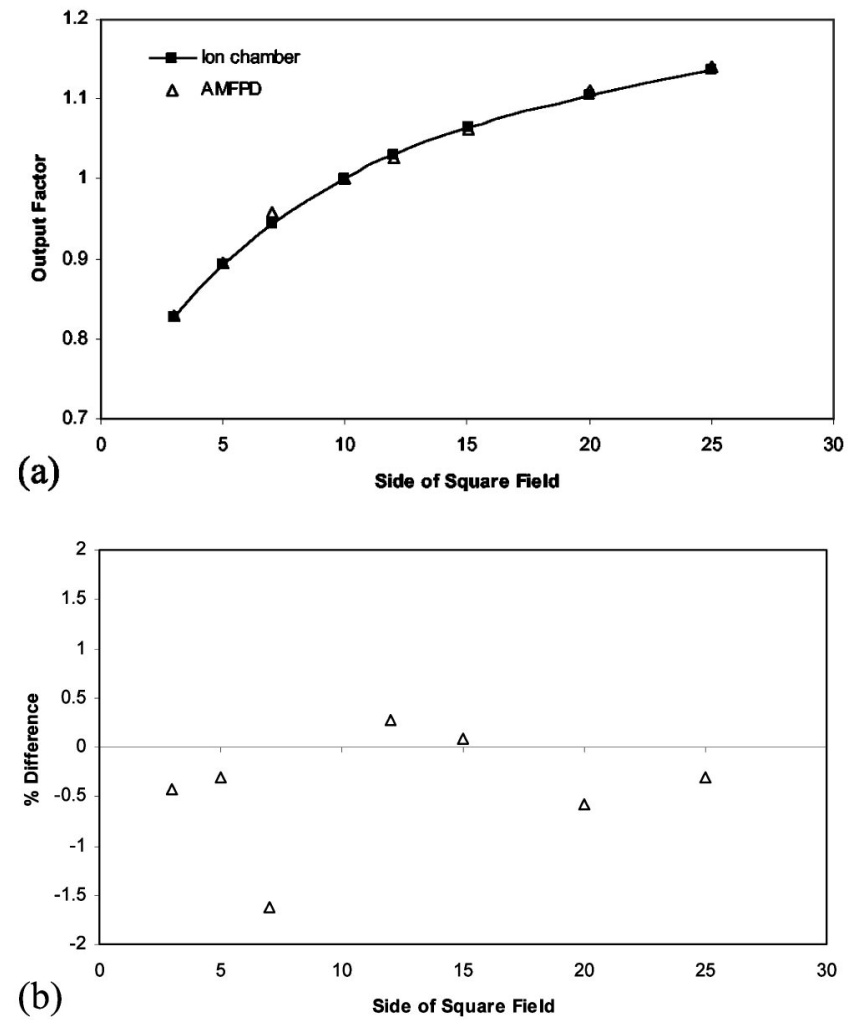

FIG. 4. (a) A comparison of the field size dependence of the output factor for ion chamber (squares) and AMFPD (triangles) measurements in solid water. (b) The relative difference (in percent) between the ion chamber and AMFPD data shown in (a).

The dosimetric response of the system was evaluated for dose, dose rate and field size. A comparison of the calibration measurements for 6 and $16 \mathrm{MV}$ were found to agree to within $3 \%$. The dependence of the system on the dose rate was measured for $6 \mathrm{MV}$ and found to be less than $1 \%$ for all dose rates. To evaluate the dependence of the AMFPD on field size, the output factor was determined relative to a 10 $\times 10 \mathrm{~cm}^{2}$ field for square field sizes ranging from 3 $\times 3 \mathrm{~cm}^{2}$ to $25 \times 25 \mathrm{~cm}^{2}$ [Fig. 4(a)]. The AMFPD results were within $2 \%$ of the ion chamber measurements [Fig. 4(b)].

The AMFPD system was developed for measurement of in-phantom planar dose distributions. Figure 5(a) shows a 2-D dosimetric comparison for a $6 \mathrm{MV} 10 \times 10 \mathrm{~cm}^{2}$ field measured with film and the AMFPD at $10 \mathrm{~cm}$ depth normalized to $100 \%$ dose at the central axis of the field. Agreement is within $2 \%$ between the isodose lines (except for the penumbra region). Figure $5(\mathrm{~b})$ shows a profile extracted from both images across the center of the field. In addition, an ion chamber $\left(0.13 \mathrm{~cm}^{3} \mathrm{CC} 13\right.$ chamber $)$ profile is shown, measured under similar conditions (except in a water phantom). Agreement between the ion chamber and AMPFD data is within $1 \%$ in the majority of the field. Some larger differences are seen in the penumbra region, where averaging over the ion chamber volume results in a less accurate dose determination in the penumbra region. ${ }^{16}$ Figure 6 shows a com- 

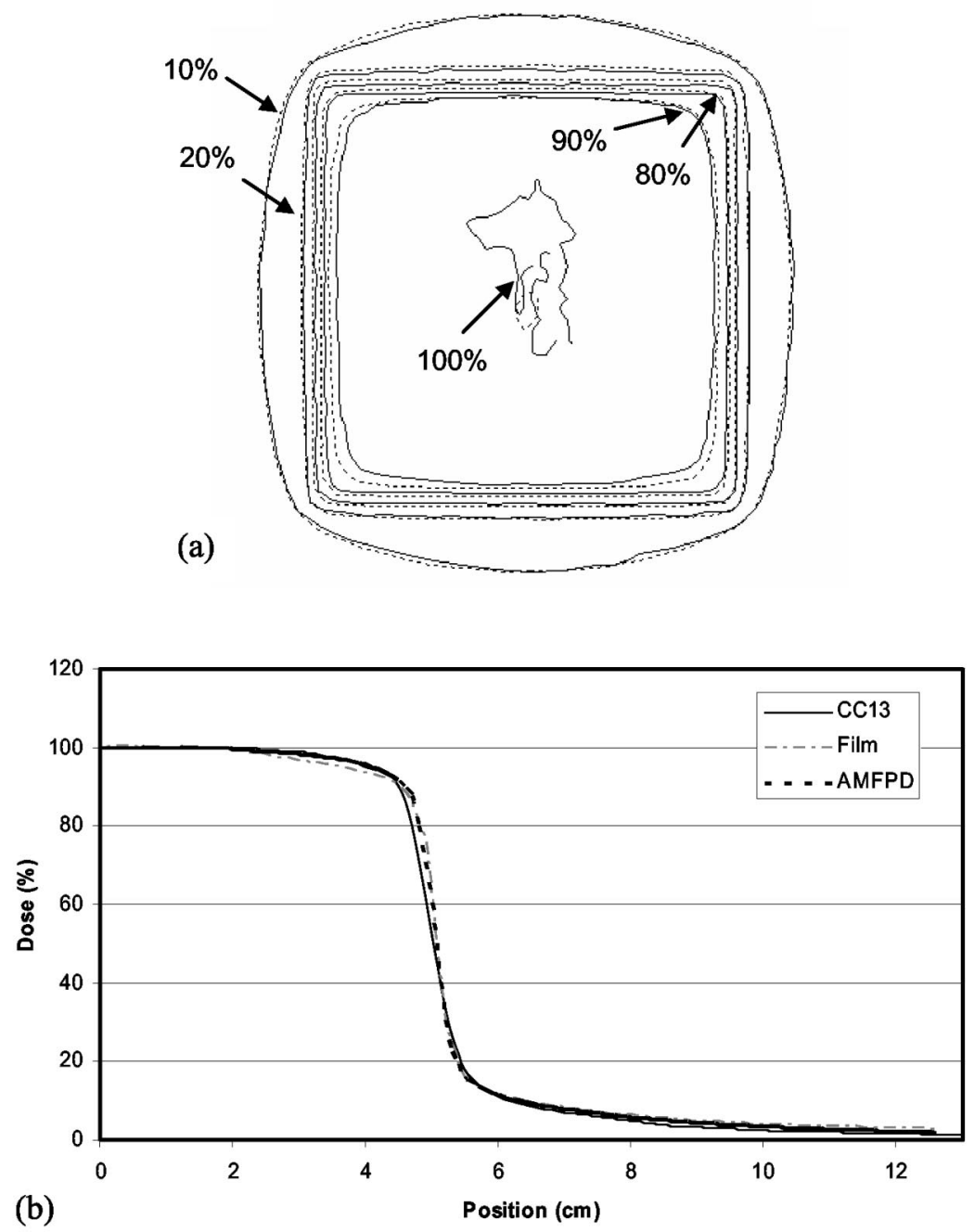

FIG. 5. (a) The planar dose distribution comparison for a $10 \times 10 \mathrm{~cm}^{2}$ field at $10 \mathrm{~cm}$ depth for film (solid lines) and AMFPD (dotted lines) in solid water. Isodose lines of $100 \%, 90 \%, 80 \%, 50 \%, 20 \%$, and $10 \%$ are shown. (b) Extracted field profiles across the central axis from film (dashed-dotted line) and AMFPD (dashed line) are compared to ion chamber measurements (solid line) in water. parison between the film and AMFPD data for a field shaped as a "C" with an MLC at depths of 1.5 and $10 \mathrm{~cm}$ normalized at $x=-4 \mathrm{~cm}, y=0 \mathrm{~cm}$. Good agreement is seen for the isodose lines displayed. At $1.5 \mathrm{~cm}$ depth, there is a discrepancy in the $100 \%$ isodose curve. At $10 \mathrm{~cm}$ depth, there are some discrepancies seen in the $10 \%$ isodose lines.

Finally, a sample SMLC-IMRT field was measured at 5 $\mathrm{cm}$ depth. Figure 7 shows a dosimetric comparison of the film and AMFPD measurements in cGy. The film and AMFPD data were converted to dose using the appropriate calibration curves without renormalization. Excellent agreement was seen for most isodose lines. Small discrepancies between lines within the field represent differences in dose up to approximately $5 \mathrm{cGy}$.

\section{DISCUSSION}

A prototype $a$-Si Active Matrix Flat Panel Imager (AMFPI) has been successfully modified for use as an in-phantom 2-D dosimetry system, guided by an earlier investigation. ${ }^{14}$ The resulting Active Matrix Flat Panel Dosimeter (AMFPD) system has been shown to be useful across a wide range of delivered doses (up to $160 \mathrm{cGy}$ for an individual irradiation) and maintains a stable calibration over a period of a year. Further work using the AMFPD for beam commissioning and IMRT measurements is underway.

The AMFPD response is independent of dose rate, to better than $\pm 1 \%$, for dose rates up to $480 \mathrm{cGy} / \mathrm{min}$. The absence of dose rate dependence is very important for any dosimetric system, especially for applications involving the dosimetric verification of IMRT fields where the actual dose rate at the AMFPD may vary during delivery. The AMFPD calibration and sensitivity appear to be very stable. While the original prototype imager system was shown to be stable for a period of over 2 months, ${ }^{14}$ the present work indicates that the calibration curve of the AMFPD varies by less than $1.5 \%$ over a full 12 month period. In addition, output factors, measured with the AMFPD, agreed to within $2 \%$ of ion chamber measurements, extending the applicability of the device for commissioning measurements. Finally, the preamplification coefficients have been stable with time. Therefore, it is not necessary to recalibrate the preamplifiers before each measurement, although the coefficients are nevertheless monitored on a regular basis.

There are a number of potential issues for the practical use of such AMFPD systems which require further investi- 


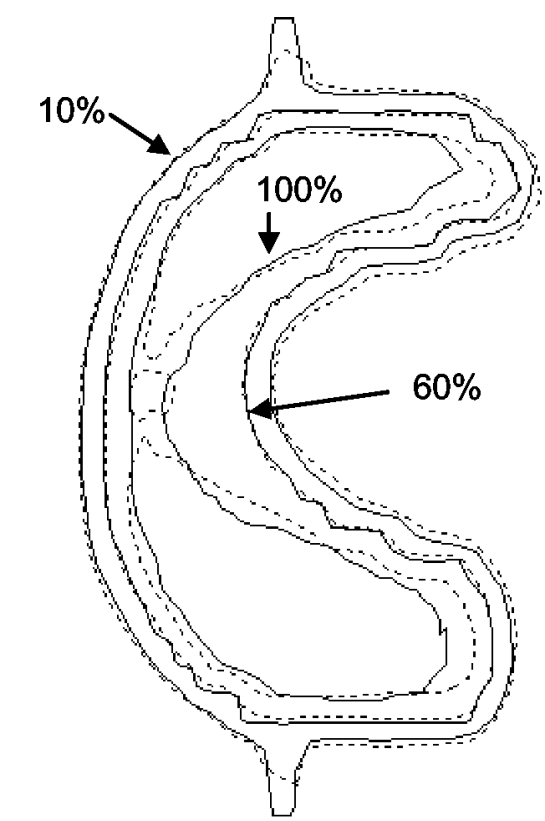

(a)

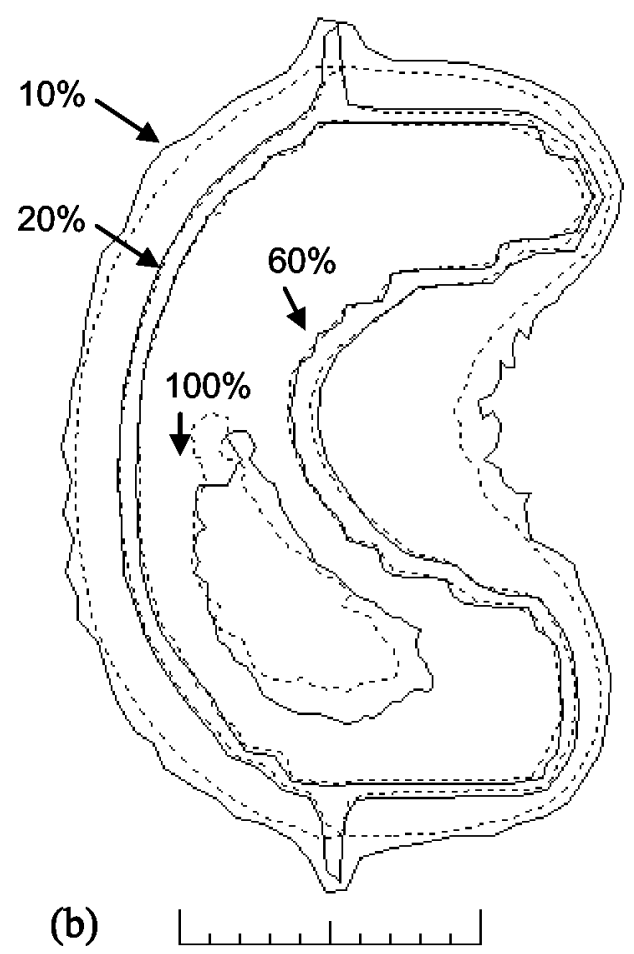

FIG. 6. Planar dose distribution comparisons for a shaped field for film (solid lines) and AMFPD (dotted lines) at (a) $1.5 \mathrm{~cm}$ depth for isodose lines of $100 \%, 60 \%$, and $10 \%$ and (b) $10 \mathrm{~cm}$ depth for isodose lines of $100 \%$, $60 \%, 20 \%$, and $10 \%$. The scale is in $1 \mathrm{~cm}$ increments.

gation. The current method of correcting for pixel-to-pixel variations requires a specific sequence of AMFPD operation. Prior to irradiation and measurement of the field of interest, dark frames are acquired and then a flood field is delivered and measured. In this calibration process, the dark frames are assumed to be free from residual signal contributions from

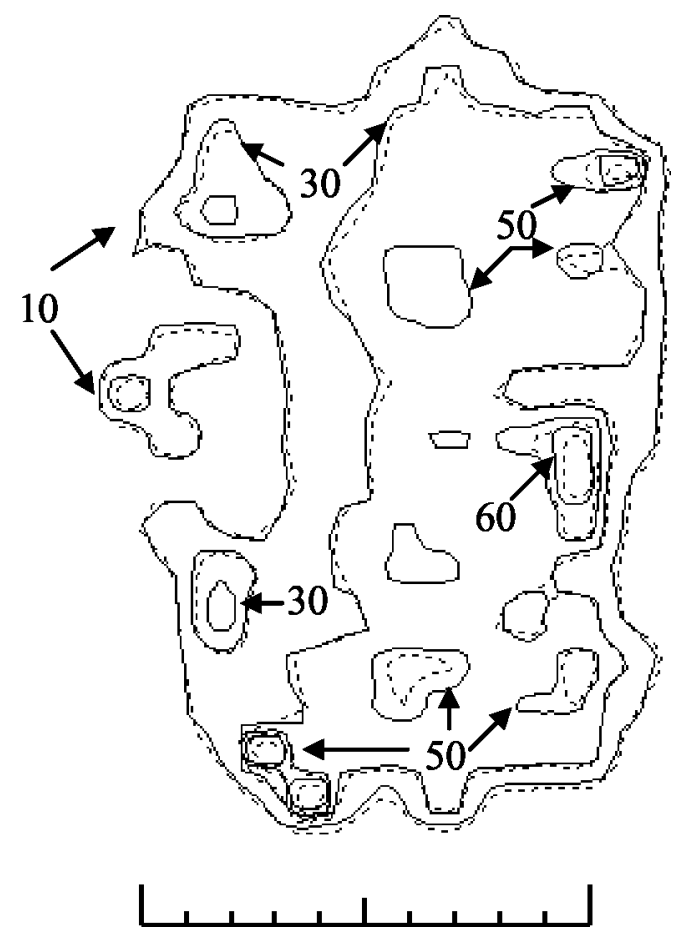

FIG. 7. Planar dose comparison for an SMLC-IMRT field at $5 \mathrm{~cm}$ depth for film (solid) and AMFPD (dotted) in solid water. Isodose lines of 60, 50, 30, and $10 \mathrm{cGy}$ are shown. The scale is in $1 \mathrm{~cm}$ increments.

previous radiation exposures. However, due to the high doses used in this system, such contributions, in the form of ghosting and lag, may affect the accurate determination of dark frames. ${ }^{15,17}$ The effects of ghosting and lag on a given measurement can be accentuated for IMRT fields due to the potential range of intensities in a given field. Hence, solutions must be devised for clinical applications.

When evaluating the dose response outside the field, it should be noted that the AMFPD over-responds to lowenergy photons due to the atomic number of silicon and other materials in the system, similar to the over-response seen with film and diodes. ${ }^{18}$ The impact of this difference on IMRT fields depends on the delivery method of the field. A Monte Carlo evaluation of transmission through the MLC demonstrated the hardening of photon beams after transport through a multileaf collimator. ${ }^{19}$ Depending on the complexity of the IMRT field, leakage can comprise as much as $10 \%$ of the maximum in-field dose. ${ }^{19,20}$ This leads to a decrease in the number of low-energy photons reaching the film or AMFPD device. For dynamic delivery, it was determined that there is an increased scatter dose through the curved leaf tips. ${ }^{19}$ Therefore, this effect would need to be considered when evaluating film or AMFPD measurements of such fields.

Clear advantages of the AMPFD system over film dosimetry include the electronic acquisition of dosimetric data and automatic data processing. In addition, the AMPFD is ideal for technique comparisons at a single depth because the alignment of the beam to the detector is known and is constant for multiple measurements. In contrast, film dosimetry requires fiducials to determine the position of each film with 
respect to the beam and a new film must be placed in the phantom for each measurement. For each experiment, a dosimetric characteristic response curve must be measured to account for variations in film processing. The number of films required for the acquisition of the characteristic curve can be reduced by using a method with multiple dose levels per film. ${ }^{21}$ After data acquisition, each film must be developed and digitized. Finally, it must be independently registered with film analysis software and converted to dose for comparison to calculations or other data. A reliable film dosimetry program also requires QA of the processor and digitizer.

While the AMFPD was intended specifically to replace film for measurements at multiple depths in a phantom, some limited comparisons can be made to commercial AMFPIs that have been investigated for dosimetry. ${ }^{6,722-24}$ In those studies, commercial AMFPI systems (aS500, Varian Medical Systems, Palo Alto, CA) were operated in a continuousacquisition mode during dose delivery and the response was then averaged over all frames in order to limit saturation of the imager pixels. ${ }^{7}$ When operating in that mode, some loss of signal occurs due to dead time while the frames are read out by the acquisition electronics. ${ }^{24}$

Because AMFPI systems include scintillator material above the detector, a water-equivalent depth of approximately $8 \mathrm{~mm}$ has been determined experimentally for the commercial AMFPI aS500 system. ${ }^{7}$ Models have been developed to address the effect of the scintillator on EPID response. For example, energy deposition in the detector has been modeled using dose kernels to generate a detector response function. ${ }^{6}$ Another approach models the detector response by deconvolving the raw image with (1) a Monte Carlo-calculated scatter kernel for dose deposition in the scintillator and (2) an empirically-derived kernel for optical photon spreading. ${ }^{23}$ For use with a pencil beam model for dose calculation, improved agreement between calculations and EPID response was shown when the portal dose was calculated in the AMPFI and not in water. ${ }^{7}$

The role of the AMPFD differs significantly from commercial AMFPIs. The effect of the scintillator as well as the difficulties in using buildup for gantry-mounted systems (due to weight limits on the control arm), limit the application of current commercial AMFPIs primarily to single plane verification such as that required for IMRT pre-treatment QA. As such, commercial AMFPIs offer a significant time-savings over current pre-treatment verification methods. The AMFPD described in this work is much more similar to a film dosimetry replacement in solid water, due to the absence of a fluorescent screen, compared to commercial AMFPIs. The system has the potential to replace film dosimetry at a time when many radiation oncology departments are considering removing film processors because EPIDs are used for all patient positioning verification.

\section{CONCLUSIONS}

A prototype $a$-Si imaging system has been successfully modified to perform dosimetric measurements in solid water.
The active matrix flat panel dosimeter (AMFPD) system described in this paper differs from commercial AMFPI portal imaging systems in a number of respects. The system operates in direct detection mode, without a fluorescent screen. The electronics associated with the system have been modified to give it an increased dynamic range so that up to 160 cGy can be measured in a single integration. The AMFPD is mounted in a frame and backed with solid water, enabling in-phantom measurements at multiple depths. The dose calibration curve of the system is stable. Comparisons to ion chamber and film measurements for sample static-MLC and IMRT field shapes show the potential of the AMFPD as a dosimeter.

Future work will characterize, in detail, the use of the AMFPD for beam commissioning and IMRT quality assurance measurements. With additional effort, it is expected that the AMFPD will prove to be an essential dosimeter for 3-D and IMRT dose calculation verification and machine commissioning data.

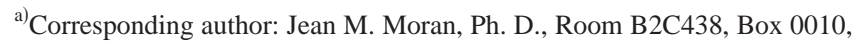
1500 East Medical Center Dr., Ann Arbor, Michigan 48109-0010. Phone: 734-936-2062; fax: 734-936-2261; electronic mail: jmmoran @ med.umich.edu

${ }^{1}$ M. C. Kirby and P. C. Williams, "The use of an electronic portal imaging device for exit dosimetry and quality control measurements," Int. J. Radiat. Oncol., Biol., Phys. 31, 593-603 (1995).

${ }^{2}$ K. L. Pasma et al., "Accurate portal dose measurement with a fluoroscopic electronic portal imaging device (EPID) for open and wedged beams and dynamic multileaf collimation," Phys. Med. Biol. 43, 20472060 (1998).

${ }^{3}$ S. N. Boon et al., "Performance of a fluorescent screen and CCD camera as a two-dimensional dosimetry system for dynamic treatment techniques," Med. Phys. 27, 2198-2208 (2000).

${ }^{4}$ Y. Zhu, X. Q. Jiang, and J. Van Dyk, "Portal dosimetry using a liquid ion chamber matrix: dose response studies," Med. Phys. 22, 1101-1106 (1995).

${ }^{5}$ H. Keller, M. Fix, and P. Ruegsegger, "Calibration of a portal imaging device for high-precision dosimetry: a Monte Carlo study," Med. Phys. 25, 1891-1902 (1998).

${ }^{6}$ B. M. McCurdy, K. Luchka, and S. Pistorius, "Dosimetric investigation and portal dose image prediction using an amorphous silicon electronic portal imaging device," Med. Phys. 28, 911-924 (2001).

${ }^{7}$ A. Van Esch, T. Depuydt, and D. P. Huyskens, "The use of an $a$ Si-based EPID for routine absolute dosimetric pre-treatment verification of dynamic IMRT fields," Radiother. Oncol. 71, 223-234 (2004).

${ }^{8}$ J. R. Symonds-Tayler, M. Partridge, and P. M. Evans, "An electronic portal imaging device for transit dosimetry," Phys. Med. Biol. 42, 22732283 (1997).

${ }^{9}$ K. L. Pasma et al., "Transit dosimetry with an electronic portal imaging device (EPID) for 115 prostate cancer patients," Int. J. Radiat. Oncol., Biol., Phys. 45, 1297-1303 (1999).

${ }^{10} \mathrm{X}$. He et al., "Evaluation of an electronic portal imaging device for transit dosimetry," Acta Oncol. 38, 591-596 (1999).

${ }^{11}$ E. E. Grein, R. Lee, and K. Luchka, "An investigation of a new amorphous silicon electronic portal imaging device for transit dosimetry," Med. Phys. 29, 2262-2268 (2002).

${ }^{12}$ M. Partridge, J. R. Symonds-Tayler, and P. M. Evans, "IMRT verification with a camera-based electronic portal imaging system," Phys. Med. Biol. 45, N183-196 (2000)

${ }^{13}$ M. Partridge, M. Ebert, and B. M. Hesse, "IMRT verification by threedimensional dose reconstruction from portal beam measurements," Med. Phys. 29, 1847-1858 (2002)

${ }^{14}$ Y. El-Mohri et al., "Relative dosimetry using active matrix flat-panel imager (AMFPI) technology," Med. Phys. 26, 1530-1541 (1999).

${ }^{15} \mathrm{D}$. A. Roberts et al., "Charge trapping at high doses in an active matrix flat panel dosimeter," IEEE Trans. Nucl. Sci. 51, 1427-1433 (2004). 
${ }^{16}$ W. U. Laub and T. Wong, "The volume effect of detectors in the dosimetry of small fields used in IMRT," Med. Phys. 30, 341-347 (2003).

${ }^{17}$ J. H. Siewerdsen and D. A. Jaffray, "A ghost story: spatio-temporal response characteristics of an indirect-detection flat-panel imager," Med Phys. 26, 1624-1641 (1999).

${ }^{18} \mathrm{M}$. Westermark et al., "Comparative dosimetry in narrow high-energy photon beams," Phys. Med. Biol. 45, 685-702 (2000).

${ }^{19}$ J. O. Kim et al., "A Monte Carlo study of radiation transport through multileaf collimators," Med. Phys. 28, 2497-2506 (2001).

${ }^{20} \mathrm{R}$. Mohan et al., "The impact of fluctuations in intensity patterns on the number of monitor units and the quality and accuracy of intensity modu- lated radiotherapy," Med. Phys. 27, 1226-1237 (2000).

${ }^{21}$ N. L. Childress, L. Dong, and Rosen, II, "Rapid radiographic film calibration for IMRT verification using automated MLC fields," Med. Phys. 29, 2384-2390 (2002).

${ }^{22}$ J. Chang and C. C. Ling, "Using the frame averaging of aS500 EPID for IMRT verification,” J. Appl. Clin. Med. Phys. 4, 287-299 (2003).

${ }^{23} \mathrm{~B}$. Warkentin et al., "Dosimetric IMRT verification with a flat-panel EPID," Med. Phys. 30, 3143-3155 (2003).

${ }^{24}$ P. B. Greer and C. C. Popescu, "Dosimetric properties of an amorphous silicon electronic portal imaging device for verification of dynamic intensity modulated radiation therapy," Med. Phys. 30, 1618-1627 (2003). 